\title{
Numerical Prediction of Microbubble Attachment in Biological Flows
}

\author{
Joshua Gosney* and Jeffrey J. Heys \\ Department of Chemical and Biological Engineering, Montana State University, Bozeman, MT \\ Student:joshgosney@gmail.com* \\ Mentor:jeffrey.heys@coe.montana.edu
}

\begin{abstract}
Biofilm infections pose a major threat to human health and are difficult to detect. Microbubbles provide an effective and inexpensive method of detection for biofilm-based infections and other diseases such as cancer. The approach studied here examines the potential of targeted microbubbles, with specific antibodies covalently linked to their surfaces for use as ultrasound contrast agents and drug delivery vehicle. This work presents a novel numerical model for estimating the forces on microbubble conjugates in the vascular system. A full computational fluid dynamics simulation of biological fluid flow and the resulting forces on attached microbubbles is presented as well as comparisons with simplified analytical models. Both the computational and analytical predictions are compared with experimental measurements from Takalkar et al. and Schmidt et al., and these comparisons indicate stable microbubble attachment can be anticipated when the total hydrodynamic force on the microbubble is less than $100 \mathrm{pN}$. Through the examination of typical biological flows, microbubble attachment can be expected up to an average fluid velocity of $0.025 \frac{\mathrm{cm}}{\mathrm{s}}$ near the microbubble (i.e., a particle Reynolds number on the order of .001). The Stokes drag law was shown to predict the drag force (the dominant force) on the microbubble within an order of magnitude of the force predicted by the numerical model. Finally, it was found that the lift force on a microbubble was small relative to the drag force, and that the Saffman equation prediction differed from the numerical model by more than an order of magnitude for the biological flows examined.
\end{abstract}

\section{KEYWORDS}

Microbubble Attachment; Ultrasound Contrast Agent; Hydrodynamic Force; Computational Fluid Dynamics

\section{INTRODUCTION}

The NIH estimates that $80 \%$ of all microbial infections are biofilms, ${ }^{1}$ which pose a significant threat to human health. The additional expenditures caused by biofilm infections are estimated to be $\$ 0.3-\$ 2.3$ billion a year. ${ }^{2,3}$ The current method for determining the presence of a biofilm-based infection on a medical device or tissue is via microscopy through the use of a scanning electron or optical microscope. While highly accurate, this method of detection is very invasive, usually requiring the removal and destruction of an implanted device or tissue. In a study done by Passerini, 4 81\% of indwelling catheters showed evidence of a biofilm on the surface of the device, but a site swab taken at the dermal entry point only detected bacteria $6 \%$ of the time. A potential alternative approach, recently demonstrated through an in vitro experiment, ${ }^{5}$ utilizes targeted microbubbles as a contrast agent for detecting and imaging biofilm infections. This method of detection would be limited to the outside surface of the catheter as a large acoustic impedance mismatch would disrupt detection of biofilms on the inner catheter surface. The microbubbles bind to the biofilm due to antibodies that are bound to their surface, and the microbubbles are imaged using ultrasound due to the density difference between biological fluids and the gas filled microbubbles. The potential advantages of this system for the detection of biofilm infections include lower costs, avoiding invasive medical device removal, and the potential for earlier biofilm detection.

These same microbubbles targeted at the biofilm could also potentially be used as a treatment delivery platform. Experiments conducted in vivo have shown that microbubble destruction via sonication is both an effective delivery method for the delivery of therapy agents and microbubble rupture during sonication could be enough to break up the biofilm. Targeted drug delivery to a location adjacent to the targeted tissue is often sufficient because the therapeutic drugs are often capable of diffusing to the target cells and tissues. ${ }^{6,7}$ Even without microbubbles, focused ultrasound energy can disrupt and fracture tissue or biofilms, a technique known as histotripsy. ${ }^{8,9}$ For any of these treatments to be utilized, the biofilm must first be detected. 
Conjugated microbubbles were first developed in the 1990s and have provided a new avenue for medical imaging that has not been available in the past. ${ }^{10-13}$ The combination of ultrasound imaging techniques and targeted microbubbles is becoming more effective than ever before at detecting diseases that express a target that the microbubbles can bind. ${ }^{10}, 11$ Persistent microbial infections, carcinomas, autoimmune disorders and amyloid plaques have proven to be difficult to detect and treat due to the toxicity and limited effectiveness of most current allopathic treatments. ${ }^{14} \mathrm{~A}$ wide range of antibodies are commercially available that can be covalently linked to microbubbles and injected into the bloodstream to detect relevant disease targets and deliver therapeutic agents. For example, it has previously been shown that amyloid plaques could be targeted using biotinylated microbubbles ${ }^{11}$, squamous cell carcinomas can be targeted with Bleomycin microbubbles ${ }^{7}$, and microbubbles can be targeted to the regulatory receptors that cause Crohn's disease. ${ }^{15}$ Targeted microbubbles have also been experimentally tested in a flow chamber with results indicating that microbubbles have a predictable, target specific attachment frequency that is a function of the flow rate. ${ }^{17-17}$ The experiments conducted by Takalkar et al. involved flowing microbubbles targeted to P-selectin through a flow chamber with varying surface densities of P-selectin. The principle result of this paper was that attachment and microbubble accumulation depended strongly on the fluid shear stress and the density of target receptors. Schmidt et al. conducted a very similar experiment, using a NeutrAvidin-coated polystyrene substrate. The observations of this paper indicated that shear from the flow was a major factor in microbubble binding and reversible binding caused by low intensity ultrasound could disrupt binding events. Further, they showed high acoustic pressure could cause microbubble destruction and therapeutic agent delivery.

Contrast agent implementations have been researched for the detection and treatment of carcinomas and atherosclerotic plaque, but little research has been conducted on the detection of biofilm infections using microbubbles. ${ }^{12,}$ 18-22 Experiments have shown that microbubbles will bind specifically to $S$. aureus in a closed system, but no estimates have been made about the behavior and attachment potential for microbubbles under common biological flow conditions.

There are two primary objectives for this work. The first objective is to develop a FEM model that estimates the bydrodynamic forces that a microbubble is exposed and validate the model by comparing its prediction of microbubble attachment to targets with experimental measurements of microbubble adhesion under specific, controlled flow conditions. The second objective is to extend the validated FEM model to examine the potential for microbubble attachment under various biological flow conditions. Finally, the force obtained from the FEM-based numerical model are compared to simple analytical approximations of the force for isolated spheres in idealized fluid flow conditions. The environment of a microbubble in the body is somewhat different from where the analytical expressions are expect to be valid, but the FEM model is used to assess the potential accuracy of the simple, analytical approximation of the force for typical biological flows.

\section{METHODS AND PROCEDURES}

For bovine serum albumin (BSA) shelled microbubbles, which are a commonly used type of microbubbles, the average shear modulus for the shell has been estimated to be in the range of $6.6 \mathrm{MPa}$ to $16.9 \mathrm{MPa}$, depending on the microbubble diameter. ${ }^{23}$ Based on this estimate and stress-strain calculations for a spherical shell, the microbubbles were assumed to be spherical and rigid for the flow conditions and forces of interest within this paper. The fluids were assumed to be incompressible, Newtonian fluids.

For the geometries and flows of interest here, the microbubbles are assumed to be attached to a surface, and the primary direction of flow is parallel to that surface. Under these conditions, only two components of the three-dimensional force vector were nonnegligible: one component is parallel to the direction of flow (the drag force, Figure 1) and the other is perpendicular to the direction of flow and normal to the attached surface (the lift force). The buoyancy force was neglected as it is at least two orders of magnitude less than the other forces for normal microbubble sizes and under typical biological flow conditions of interest.

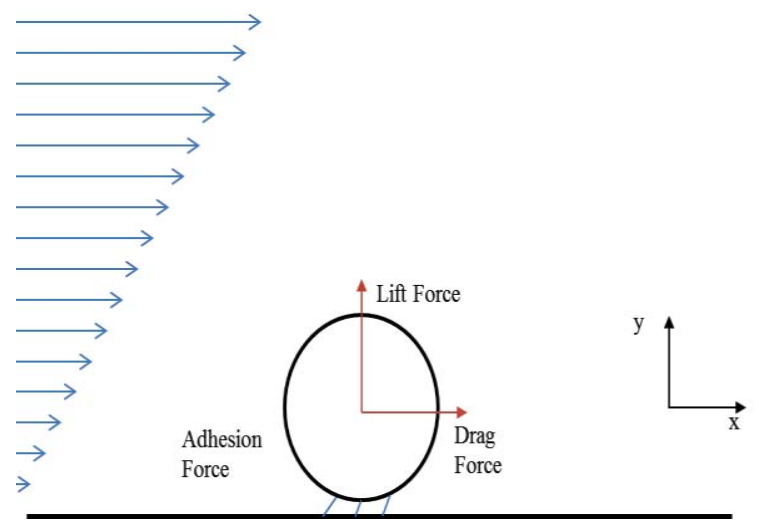

Figure 1. A microbubble attached to the surface and the forces of interest are shown in a linear flow field (not to scale). 
Numerical Model

The main objective of this research is to develop a numerical model that estimates the force on a microbubble under various biological flow conditions and compare these estimates to the analytical model. The numerical studies were conducted using the finite element method to approximately solve the Navier-Stokes equations for incompressible flow. ${ }^{24}$

$$
\begin{array}{cr}
-\nabla p+\nabla \cdot \mu\left[\left(\nabla u+\nabla u^{T}\right)\right]=\rho\left(u \cdot \nabla u+\frac{\partial u}{\partial t}\right) & \text { Equation } 1 . \\
\nabla \cdot u=0 & \text { Equation } 2 .
\end{array}
$$

where $u$ is the velocity vector, $\mu$ is the viscosity, $p$ is the pressure, and $\rho$ is the density of the fluid.

The finite element method was used for the spatial discretization of the Navier-Stokes equations, and the mesh resolutions were varied from 88,762 - 325,000 mixed finite elements (Taylor-Hood tetrahedral elements). Grid continuation studies indicated that the forces on a sphere attached to the wall could be considered relatively (less than $2 \%$ change in the drag force with refinement) grid independent for these mesh resolutions as long as the microbubble had at least 3300 elements on its surface (i.e., the mesh had to be sufficiently refined near the microbubble). As the number of elements increased, the microbubble geometry was

\begin{tabular}{|c|c|c|c|}
\hline Geometry & Dimensions & $\begin{array}{l}\text { Average inflow velocity } \\
\text { Particle Reynolds number }\end{array}$ & $\begin{array}{l}\text { Domain/ } \\
\text { microbubble size } \\
\text { ratio }\end{array}$ \\
\hline Parallel Plate ${ }^{16}$ & $0.6 \mathrm{~mm} \times 8 \mathrm{~mm}$ & $\begin{array}{l}V_{a v g}=0.2 \frac{\mathrm{cm}}{\mathrm{s}} \text { to } 1.5 \frac{\mathrm{cm}}{\mathrm{s}} \\
R e_{p}=0.00017 \text { to } 0.0013\end{array}$ & 215 \\
\hline Parallel Plate $^{17}$ & $0.25 \mathrm{~mm} \times 3.5 \mathrm{~mm}$ & $\begin{array}{c}V_{a v g}=0.0806 \frac{\mathrm{cm}}{\mathrm{s}} \text { to } 0.4 \frac{\mathrm{cm}}{\mathrm{s}} \\
R e_{p}=0.0001 \text { to } 0.0008\end{array}$ & 145 \\
\hline Capillary $^{25}$ & Diameter $=10 \mu \mathrm{m}$ & $\begin{aligned} V_{\text {peak }} & =0.0001 \frac{\mathrm{cm}}{\mathrm{s}} \text { to } 0.03 \frac{\mathrm{cm}}{\mathrm{s}} \\
R e_{p} & =1 \cdot 10^{-6} \text { to } 1.8 \cdot 10^{-4}\end{aligned}$ & 3 \\
\hline Venule $^{25}$ & Diameter $=100 \mu \mathrm{m}$ & $\begin{array}{c}V_{\text {peak }}=0.1 \frac{\mathrm{cm}}{\mathrm{s}} \text { to } 0.3 \frac{\mathrm{cm}}{\mathrm{s}} \\
R e_{p}=2.7 \cdot 10^{-4} \text { to } 8.4 \cdot 10^{-4}\end{array}$ & 36 \\
\hline $\operatorname{Vein}^{25}$ & Diameter $=1 \mathrm{~mm}$ & $\begin{array}{c}V_{\text {peak }}=1 \frac{\mathrm{cm}}{\mathrm{s}} \text { to } 5 \frac{\mathrm{cm}}{\mathrm{s}} \\
R e_{p}=1.5 \cdot 10^{-4} \text { to } 7.3 \cdot 10^{-4}\end{array}$ & 360 \\
\hline
\end{tabular}
captured more accurately, and the grid continuation studies showed that the number of elements on the boundary of the microbubble was the most important factor in determining the numerical model accuracy.

Table 1. Description of numerically assessed geometries and their flow rates.

Blood was assumed to be a Newtonian fluid with a density of $1040 \mathrm{~kg} / \mathrm{m}^{3}$ and a constant viscosity of $3.5 \times 10^{-3} \mathrm{~kg} / \mathrm{m} / \mathrm{s}$. Blood is a suspension of cells and exhibits the behavior of a shear thinning fluid, but testing with a non-Newtonian blood flow model showed negligible deviation from the simpler Newtonian model for the flow rates and geometries described here so a Newtonian model was used. Flows with higher shear rates often require a non-Newtonian viscosity model for blood. Consistent with previous experimental results, the fluid in the model had the physical properties of PBS (phosphate buffered saline) with a 
constant viscosity of $1.05 \times 10^{-3} \mathrm{~kg} / \mathrm{m} / \mathrm{s}$. The forces on the microbubble were calculated by taking an approximate integral over the surface of the microbubble.

Two computational geometries were used in this study. A parallel plate flow channel geometry is used for comparison to previously published experimental results of Schmidt et al. and Takalkar et al., and the dimensions of the model domain match as closely as possible the flow channel used in those experiments (Table 1). ${ }^{16,17}$ In addition, representative blood vessels including capillaries, veins and arteries were modeled using a straight, cylindrical geometry (Table 1). The length of the blood vessel sections used in the model ranged from 10 microns (capillaries) to $3 \mathrm{~cm}$ (vein) with internal diameters ranging from 10 microns (capillaries) to $1 \mathrm{~mm}$ (vein)..$^{25}$ Thus, even the smallest diameter cylinders were larger than the typical microbubble diameter, which vary from 3.5 to 6 microns.

Large ranges of length scales were present in some problems where the ratio of the fluid domain size to microbubble diameter exceed 100. In these cases, it was impossible to generate a finite element mesh that was both computational feasible (i.e., did not require billions of elements) and did not contain invalid elements with negative Jacobians or extremely high aspect ratios. For these problems with a large range of length scales (i.e., for all problems where the total domain size to microbubble diameter ratio is greater than 100), a multi-scale modeling technique is employed to separate the larger length scales from the smaller length scales near the microbubble. When this technique is used, the larger fluid domain is modeled first without the microbubble explicitly included in the large length scale problem because it is significantly smaller than the individual elements. Then, after the larger length scale flow problem has been solved, the solution from the large scale problem is used to obtaining boundary conditions for the microbubble scale model, which only includes the fluid near the microbubble (i.e., the fluid within approximately 20 microbubble diameters of the center of the microbubble).

The boundary conditions for the parallel plate geometries are given in the experimental comparison section below. The capillary and venule simulations employed a range of flow rates (see Table 1), and boundary conditions were set based on a Pouiseulle flow profile. Early simulations of flow in the larger blood vessels (vein) included the effects of wall elasticity and displacement on both the velocity gradients near the wall and the forces on the microbubble. These early results showed that the largest forces would be generated with a rigid wall, consistent the observations of others ${ }^{26}$, so all results shown below are based on the most difficult conditions for microbubble attachment: the rigid wall vessel.

\section{Experimental Comparisons}

The experimental measurements by others that are used here for numerical model validation were assumed to be at steady state with a fully developed flow profile. The fluid shear stress near the wall in the experimental channel can be calculated through the equation. ${ }^{16}$

$$
Q=\frac{\tau h^{2} w}{6 \mu}
$$

Equation 3.

where $\tau$ is the wall shear stress, $h$ is the channel height, and $w$ is the channel width. The velocity in the mathematical model was set so that the wall shear stress matched the experimental wall shear stress values of $0.02,0.05,0.1$ and $0.15 \mathrm{~Pa}{ }^{16}$ The shear stresses for the second set of experiments by Takalkar et al. were 0.02, 0.03, 0.06, 0.1 and $0.17 \mathrm{~Pa} .{ }^{17}$ The channel height, $h$, was 0.6 and $0.254 \mathrm{~mm}$ for the Schmidt et al. and Takalkar et al. experiments, respectively, and since the channel width in both experiments was at least an order of magnitude greater than the height, a 2-dimensional model was used for the macro-scale fluid model. (A full 3-dimensional model was used for the micro-scale model of flow around the microbubble.) The boundary conditions used to simulate the experiments (i.e., the boundary conditions for the macro-scale model) include:

- $\quad$ no-slip $(u=0)$ at the upper and lower walls,

- the normal stress in the normal direction is zero at the outlet, and

- tangential velocity of zero at the inlet and outlet.

For the micro-scale model, the solution from the macro-scale model was used to specify the velocity boundary conditions. The adhesion forces between the microbubbles and surfaces containing the targets were estimated based on previous Atomic force microscope (AFM) measurements and are used as a basis for comparisons between experiments and model predictions. ${ }^{27}$ 
Analytical Models

The drag force on a sphere in an infinite, uniform, creeping flow can be approximated by the well-known Stokes drag law equation:

$$
F_{D}=6 \pi a \mu V
$$

Equation 4.

where $a$ is the radius of the microbubble, $V$ is the characteristic velocity around the outer perimeter of the microbubble's surface, and $\mu$ is the viscosity. The Stokes drag law assumptions include low Reynolds number flow $(\operatorname{Re}<10)$, no particle-particle interactions, and an infinite body of fluid around the spherical particle. The Reynolds number for this problem is defined as: $R e=\frac{V \cdot L \cdot \rho}{\mu}$, where $V$ is characteristic velocity (defined as the mean fluid velocity), $L$ is the characteristic length (the microbubble diameter unless otherwise specified), and $\rho$ is the fluid density. ${ }^{7}$

The Saffman equation ${ }^{28}$ can be used to estimate the lift force (i.e., the force perpendicular to the primarily flow direction) on a spherical particle due to a velocity gradient:

$$
F_{L}=K V a^{2} \frac{k^{1 / 2}}{v^{1 / 2}}
$$

where $v$ is the fluid's kinematic viscosity and $k$ is the velocity gradient. Assumptions for the lift force estimate include symmetrical flow around the center of the sphere, and a semi-infinite body of fluid around the sphere, meaning that the microbubble should be surrounded by ample fluid on all sides.

\section{RESULTS AND DISCUSSION}

The numerical simulation results are divided into two sections: first the results of the parallel plate simulations are described and compared to previous experimental measurements, and then in the second section, the results of simulations in cylindrical blood vessel geometries are presented.

\section{Parallel Plate Simulations}

The first set of simulations was focused on the square channel geometry used in previous experimental observations to estimate the microbubble binding strength. Figure 2 shows the drag force predicted by the numerical simulation for a range of Reynolds number in a geometry consistent with Schmidt et al. and Takalkar et al. ${ }^{16,24}$ The range of drag force values predicted by the model was from 14 to $100 \mathrm{pN}$ for superficial velocities between $0.2 \frac{\mathrm{cm}}{\mathrm{s}}$ and $1.5 \frac{\mathrm{cm}}{\mathrm{s}}$. Schmidt et al. observed microbubble detachment at a velocity of approximately $1.5 \frac{\mathrm{cm}}{\mathrm{s}}$ for their flow cell, and the model predicts a drag force of $100 \mathrm{pN}$ under these conditions. Takalkar et al. showed a similar result, observing detachment at a superficial velocity of $0.6 \frac{\mathrm{cm}}{\mathrm{s}}$, corresponding to a drag force of $100 \mathrm{pN}$ according to the model. Thus, based on the experiments of Takalkar et al. and Schmidt $e t$ al., the model predicts microbubble attachment when the drag force is $100 \mathrm{pN}$ or less, and no attachment when the drag force is greater than $100 \mathrm{pN}$.

Recent experiments have attempted to measure the total microbubble adhesion forces through the use of an atomic force microscope (AFM). These experiments showed a median adhesion force of $93 \mathrm{pN} .{ }^{27}$ This result is consistent with the drag force estimate from the numerical simulation combined with flow cell measurements. Since the AFM measurements are believed to have measured the adhesion force of individual bonds, the implication is that each microbubble is primarily held in place by one or a few antibody bonds. This is intriguing since the surface density of the microbubbles has been estimated to be approximately 2500 antibodies per $\mathrm{m}^{2},{ }^{17}$ so there is the potential for multiple bonds between the microbubble and the target. However, the formation of many bonds would require a high density of receptors on the target and, if there were multiple bonds, their binding strengths are unlikely to be additive because just a few bonds would be expected to be under tension as described by Ward et al. ${ }^{29}$ The drag force on the upper part of the microbubble furthest from the surface attachment bonds is likely to result in a 'peeling' or 'unzipping' of individual antibody bonds from each attachment sites.

\section{Blood Flow Simulations}

The second set of results is focused on predicting whether or not microbubble attachment is likely or unlikely for a range of blood vessel sizes and a range of blood flow velocities. The goal was to evaluate a sufficient range of vessel sizes and blood flow velocities to represent most of the conditions found in the cardiovascular system. The results are focused on predicting microbubble attachment in a "worst case" scenario and thus the model predictions of attachment may be conservative. The prediction of microbubble attachment is based on three regions of the vascular system: capillaries, venules, and veins. 


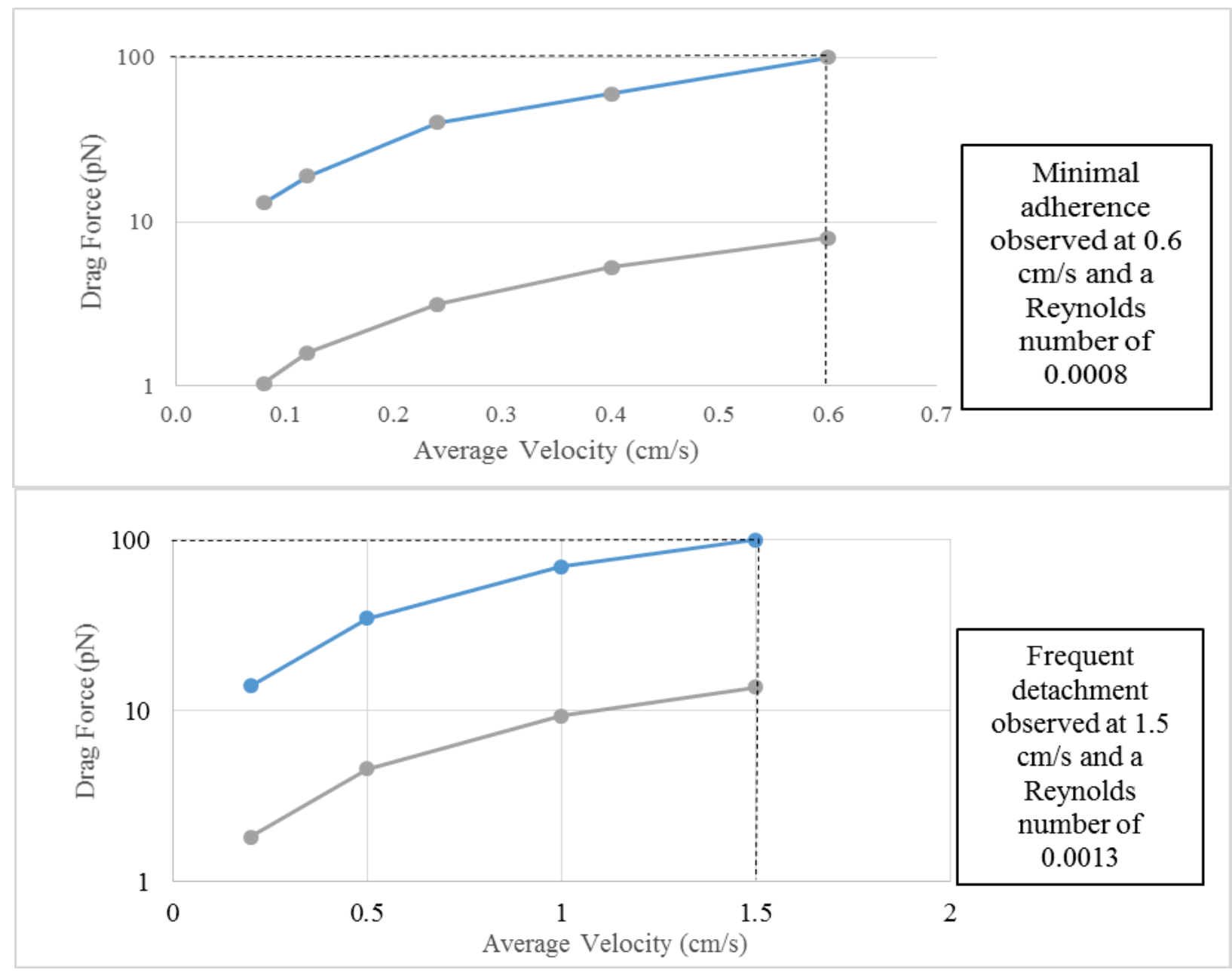

Figure 2. The numerical model predictions of drag force (blue) at different average velocities. The attachment/detachment of microbubbles based on experimental measurements by the Takalkar et al. (top) and Schmidt et al. (bottom) are indicated by the vertical dashed line. The Stokes drag law predictions of drag force are shown in gray. The model prediction of drag force combined with the experimental measurements of detachment suggest a drag force of $100 \mathrm{pN}$ results in detachment.

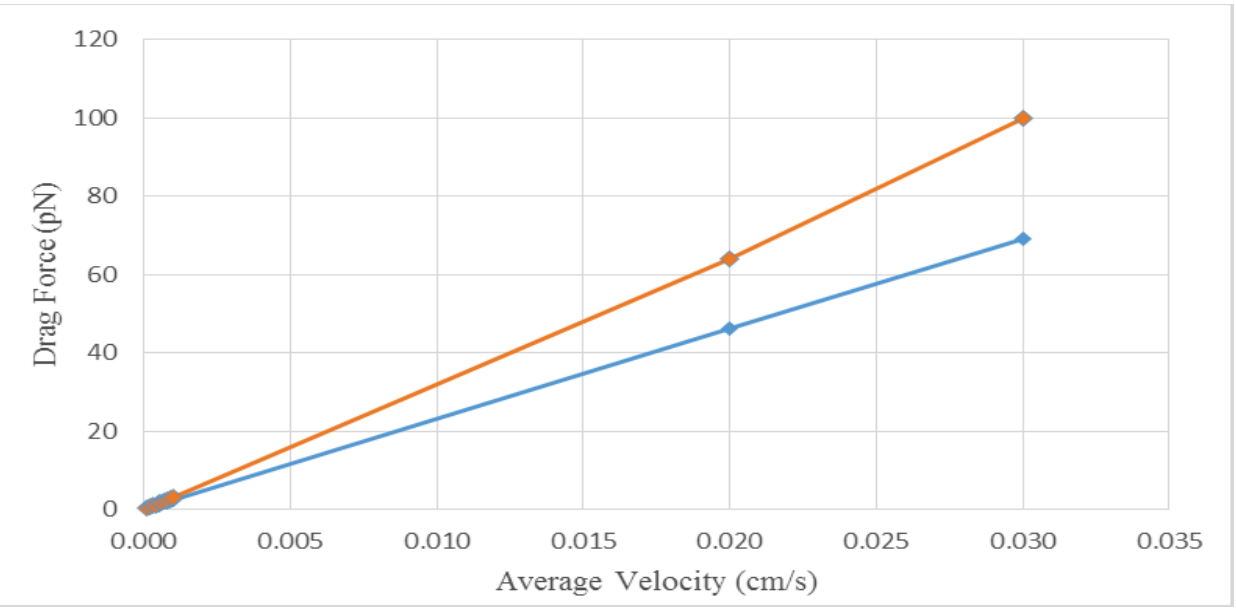

Figure 3. Numerical results (orange) from the capillary indicate that microbubbles should stay attached at all velocity. Stokes drag results (blue) are similar but diverge from the numerical results. This is most likely due the small capillary diameter to microbubble diameter ratio around .001

Additionally, these blood vessel results are compared with the predictions of the Stokes drag law. If the Stokes drag law results are consistent with the numerical model, it would allow for the use of simplified equations to predict microbubble behavior in a 
wide range of flow conditions throughout the body without the use of computational modeling software. For all simulations, the microbubble is attached to the vessel wall and the hydrodynamic forces on the bubble are estimated.

The first vessel examined is the capillary geometry, and the force increases nearly linearly with the Reynolds number over the range of flow rates examined (Figure 3). Although the total hydrodynamic forces from fluid flow are unlikely to prevent attachment based for the typical range of velocities in the capillaries (i.e., the forces are less than $100 \mathrm{pN}$ ), the additional force from the impact of red blood cells, or other small particles could be significant and potentially lead to detachment. The force predictions from the Stokes drag law differed from the more accurate numerical simulation calculation of drag by no more than $50 \%$ at low Reynolds numbers $\left(R e_{c} \ll 1\right)$ found within capillaries. The agreement between the numerical simulation and Stokes drag law is somewhat unexpected because the Stokes drag law was derived using an infinite, unbounded body of fluid. As the flow rate increases in the capillary, however, a separation between the Stokes drag and numerical results is observed and is due to the acceleration of additional fluid through the space between the microbubble and opposing wall.

The second set of numerical results address forces on the microbubble in flow regimes similar to those found in venules. It can be seen once again that the forces increase approximately linearly with velocity. In flow regimes where the velocity is less than $0.15 \frac{\mathrm{cm}}{\mathrm{s}}$, microbubble adherence should be observed for this vessel, which has a much larger diameter than the capillary.

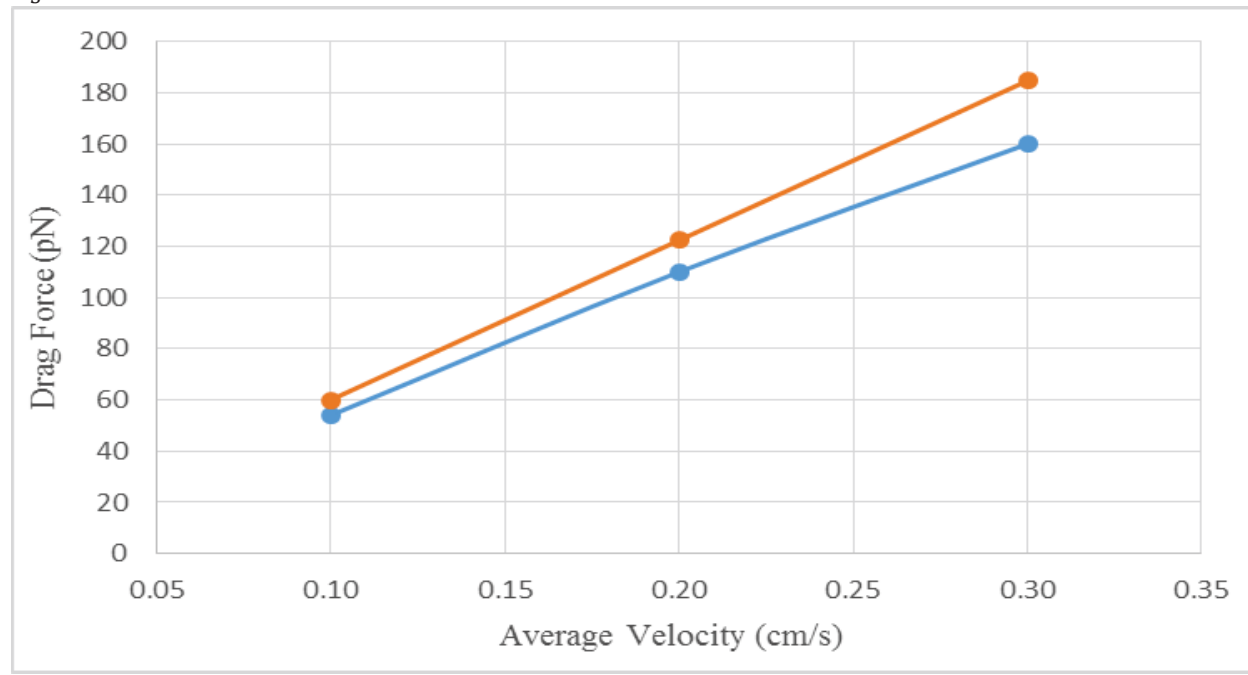

Figure 4. Numerical results in the Venuole flow regimes (orange) indicate that microbubble adherence is observed at velocities less than approximately $0.20 \mathrm{~cm} / \mathrm{s}$. The results also indicate that the Stokes drag (blue) is a relatively accurate approximation of the force within these regimes.

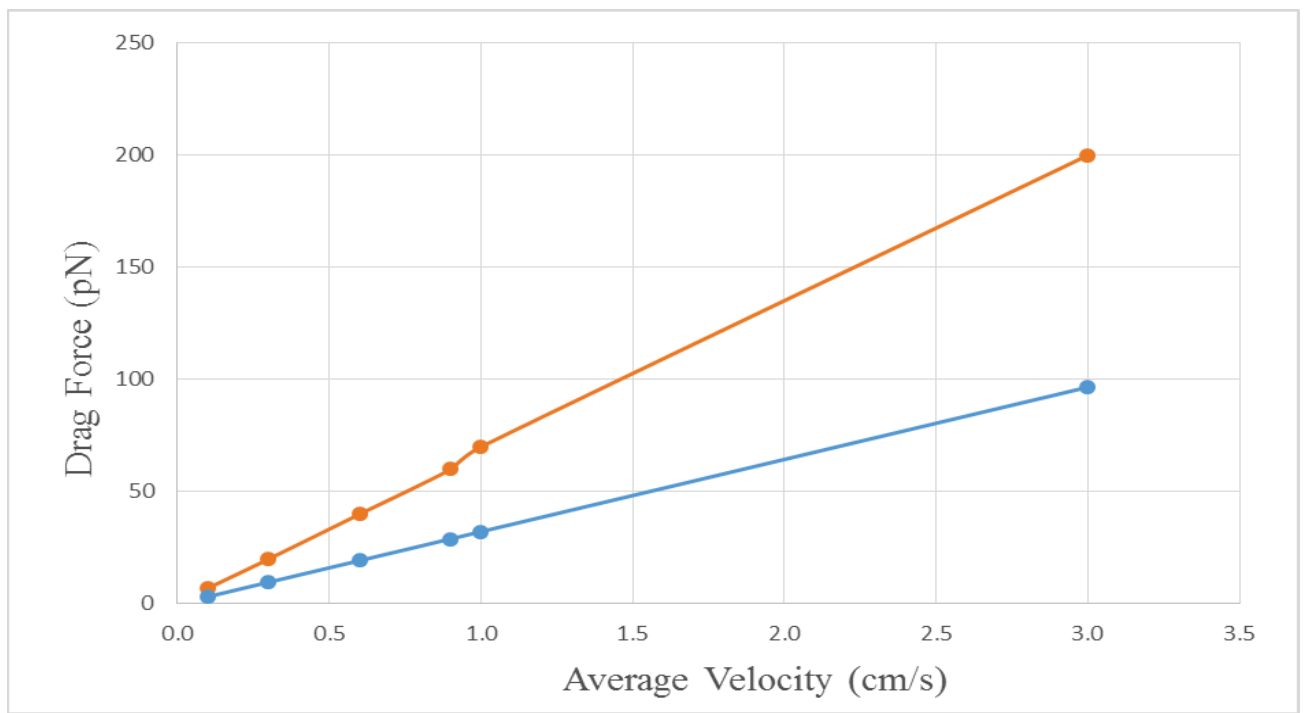

Figure 5. Numerical results from the vein flow regime (orange) indicate that microbubble attachment is observed at velocities less than $1.5 \mathrm{~cm} / \mathrm{s}$. Additionally a divergence from the Stokes drag law is observed, but the Stokes drag law was accurate within an order of magnitude for the flow regimes of interest. 
Velocities above $0.15 \frac{\mathrm{cm}}{\mathrm{s}}$ are predicted to result in frequent detachment and infrequent adherence of microbubbles. The microbubble is very small relative to the vessel size, so the microbubble is not directly in the flow path, but it does extend far enough into the main flow channel to be detached by the larger hydrodynamic forces away from the wall of the vessel. Unlike capillary flows, these vessels are much larger, so effects from red blood cells and other particles should be less pronounced. The Stokes drag law prediction of the drag force is similar to the numerical results in this regime, showing a variation of less than $10 \%$ between the two approximations (Figure 4).

The third and final set of numerical studies were conducted on the flow regimes typical for a vein. Because the microbubble is much smaller than the vein, these results were collected using the multi-scale modeling techniques described in the Methods section. The results indicate that the microbubble was likely to stay attached in areas where the average velocity in the vein was less than $1.5 \frac{\mathrm{cm}}{\mathrm{s}}$ (Figure 5). By contrast, detachment is predicted in the much smaller venule at velocities above $0.15 \frac{\mathrm{cm}}{\mathrm{s}}$. This difference is not a surprising result because the microbubble is over 1000 times smaller than a vein, but only about 50 times smaller than a venule. In other words, the microbubble is a tiny fraction of the diameter of the vein and completely out of the main fluid flow. Additionally, increasing differences between the Stokes drag law force prediction and the numerical model were observed. This is partially because the Stokes drag law can only be considered accurate at Reynolds numbers much less than one. However, the Stokes drag law is still within a factor of 2 for the flow regimes of interest, making it a potentially useful tool for predicting the overall force on a microbubble.

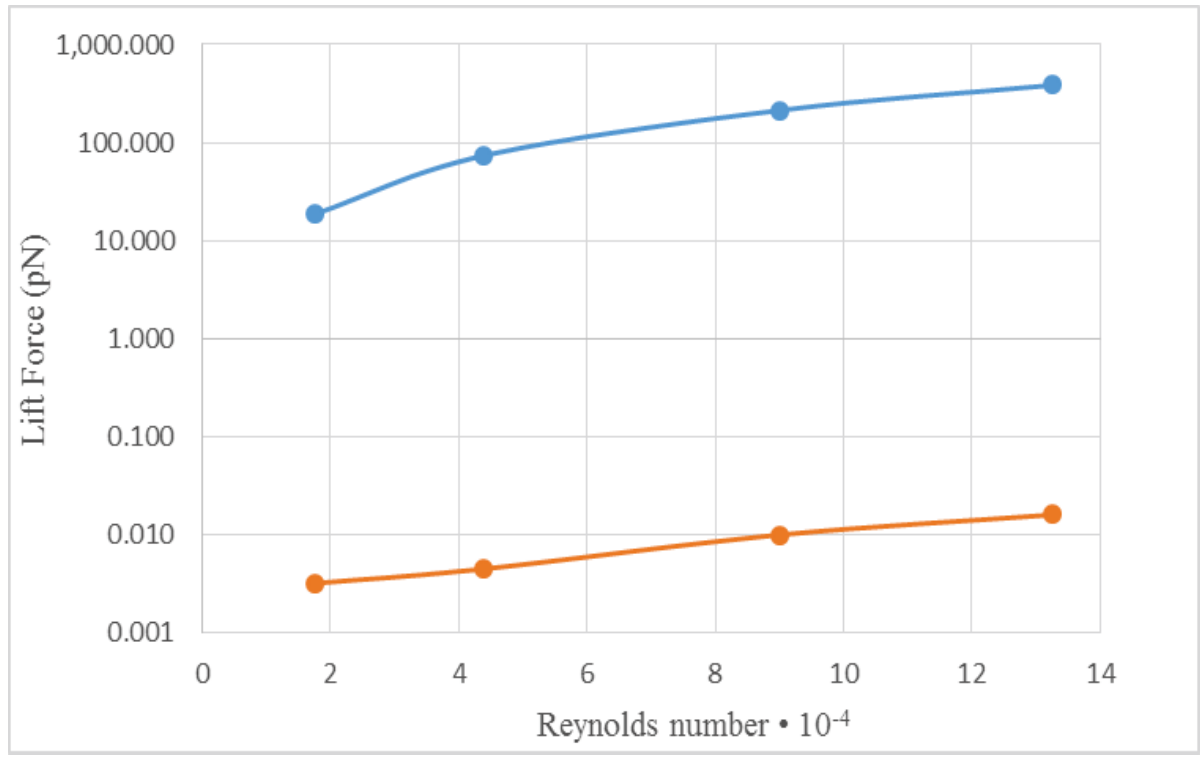

Figure 6. The numerical lift force (orange), is much smaller than the lift force predicted by the Saffman equation. This is not a surprising result, as Saffman's equation of lift is only valid when the particle is very far from the wall.

The final goal of this work was to analyze the potential effectiveness of the Saffman equation (Equation 5) for predicting hydrodynamic lift forces on the microbubble. The Stokes drag law prediction of the drag force has already been discussed and is consistently less than the numerical model results. The Saffman equation for estimating the lift force was also compared to numerical results for all of the cases outlined. The Saffman equation predicted that the lift force was consistently the same order of magnitude as the drag force, but the numerical results indicated that this was not the case (Figure 6). For all of the problem geometries and flow rates of interest, it was found that the results from the Saffman equation for predicting the lift force were at least two orders of magnitude greater than the numerical results. The Saffman equation was derived based on the assumption that the particle was in an unbounded shear flow (far from the wall), and the violation of this assumption in the problems of interest probably leads to this difference. The numerical results indicated that the lift force on the microbubble could be considered negligible for all cases of interest, displaying a lift force that was at least one order of magnitude less than the drag forces.

\section{CONCLUSIONS}

There were two primary objectives for this work. The first objective was to develop a numerical model of the forces on a microbubble in order to predict microbubble attachment/detachment, and then to validate the model based on previously published experimental measurements. The mathematical model predicted that the microbubble would adhere to its target unless it was exposed to forces greater than $100 \mathrm{pN}$. This result was consistent with AFM experiments with microbubbles that showed the median force of microbubble attachment is about $100 \mathrm{pN}$. The result suggests an unzipping effect, seen in other 
experiments ${ }^{29}$ may be important when there are multiple bonds between the microbubble and the target surface, and this effect would allow multiple antibody bonds to rupture in a sequential manner. The second objective was to develop a numerical model to predict the potential for attachment of microbubbles in various flow conditions throughout the body. The principle result from this objective is that microbubble adhesion is predicted to occur in most capillaries, some venules and some veins, depending on the velocity (or Reynolds number) of the flow.

The numerical modeling results were compared to analytical equations that predict hydrodynamic forces on spherical particles. The results indicated that the lift force predicted by the Saffman equation did not agree with the numerical model predictions, primarily because the derivation of the equation was based on the assumption that the spherical particle was in an unbounded flow (far from the wall). The results also indicated that the Stokes drag law is typically within one order of magnitude of the drag force prediction of the numerical model. Limitations of the numerical model include the assumption of a Newtonian fluid, the assumption that both the vessels and the microbubbles where rigid, and the absence of erythrocytes in the model.

Future work on this project is focused on two objectives. First, the numerical model will be extended to model therapeutic drug delivery via the target microbubbles. Using targeted drug delivery would allow not only the detection of a biofilm based infection, but would also enable the immediate delivery of either therapeutic drugs. The second future objective is additional experimental measurements to validate the microbubble attachment model, assess the effects of nearby medical device materials on the feasibility of ultrasound imaging of microbubbles, and evaluate treatment options including therapeutic drug delivery and the disruption of the physical structure of the biofilm using either ultrasound energy directly or the rupturing of microbubbles.

\section{ACKNOWLEDGEMENTS}

This work was supported by the McNair Scholars program (U.S. Dept. of Education grant \#P217A130148) and NSF grant CBET 1249950.

\section{REFERENCES}

1. Harro, J. M., Peters, B. M., O’May, G. A., Archer, N., Kerns, P., Prabhakara, R., and Shirtliff, M. E. (2010) Vaccine development in Staphylococcus aureus: taking the biofilm phenotype into consideration, Fems Immunol Med Mic 59, $306-323$.

2. Frasca, D., Dahyot-Fizelier, C., and Mimoz, O. (2010) Prevention of Central Venous Catheter-related Infection in the Intensive Care Unit, Intensive Care Medicine: Annual Update 2010, 223-234.

3. Mermel, L. A., Farr, B. M., Sherertz, R. J., Raad, I. I., O’Grady, N., Harris, J. S., and Craven, D. E. (2001) Guidelines for the management of intravascular catheter-related infections, Clin Infect Dis 32, 1249-1272.

4. Passerini, L., Lam, K., Costerton, J. W., and King, E. G. (1992) Biofilms on Indwelling Vascular Catheters, Crit Care Med 20, 665-673.

5. Anastasiadis, P., Mojica, K. D. A., Allen, J. S., and Matter, M. L. (2014) Detection and quantification of bacterial biofilms combining high-frequency acoustic microscopy and targeted lipid microparticles, J Nanobiotechnol 12.

6. Shohet, R., Chen, S., Zhou, Y. T., Wang, Z. W., Meidell, R. S., Unger, R., and Grayburn, P. A. (2000) Targeted gene delivery to the left ventricular myocardium using ultrasound-triggered microbubble destruction, J Am Coll Cardiol 35, 444a-444a.

7. Wilkes, J. O. (2006) Fluid mechanics for chemical engineers with Microfluidics and CFD, 2nd ed., Prentice Hall Professional Technical Reference, Upper Saddle River, NJ.

8. Bigelow, T. A., Northagen, T., Hill, T. M., and Sailer, F. C. (2008) Ultrasound Histotripsy and the Destruction of Escherichia Coli Biofilms, Ieee Eng Med Bio, 4467-4470.

9. Schade, G. R., Keller, J., Ives, K., Cheng, X., Rosol, T. J., Keller, E., and Roberts, W. W. (2012) Histotripsy Focal Ablation of Implanted Prostate Tumor in an ACE-1 Canine Cancer Model, J Urology 188, 1957-1964.

10. Chapuis, J. C., Schmaltz, R. M., Tsosie, K. S., Belohlavek, M., and Hecht, S. M. (2009) Carbohydrate Dependent 'Targeting of Cancer Cells by Bleomycin-Microbubble Conjugates, J Am Chem Soc 131, 2438.

11. Rand, A., Gilman, G., Kane, D., and Belohlavek, M. (2008) Biotinylated Microbubbles Targeted to Amyloid., The Open Clinical Chemistry Journal 1, 75-78.

12. Ferrara, K., Pollard, R., and Borden, M. (2007) Ultrasound microbubble contrast agents: Fundamentals and application to gene and drug delivery, Annual Review of Biomedical Engineering 9, 415-447.

13. Bloch, S. H., Dayton, P. A., and Ferrara, K. W. (2004) Targeted imaging using ultrasound contrast agents, Ieee Eng Med Biol 23, 18-29.

14. Brady, R. A., Leid, J. G., Kofonow, J., Costerton, J. W., and Shirtliff, M. E. (2007) Immunoglobulins to surface-associated Biofilm Immunogens provide a novel means of visualization of methicillin-resistant Staphylococcus aureus Biofilms, Applied and Environmental Microbiology 73, 6612-6619.

15. Brobst, T., Rombola, C., Ungerleider, S., Voncannon, S., and Ward, B. (2013) Targeted Microbubble Drug Delivery in an Experimental Treatment Model of Crohn's Disease, The Spectra, 27-35. 
16. Schmidt, B. J., Sousa, I., van Beek, A. A., and Bohmer, M. R. (2008) Adhesion and ultrasound-induced delivery from monodisperse microbubbles in a parallel plate flow cell, J Control Release 131, 19-26.

17. Takalkar, A. M., Klibanov, A. L., Rychak, J. J., Lindner, J. R., and Ley, K. (2004) Binding and detachment dynamics of microbubbles targeted to P-selectin under controlled shear flow, J Control Release 96, 473-482.

18. Stride, E. P., and Coussios, C. C. (2010) Cavitation and contrast: the use of bubbles in ultrasound imaging and therapy, P I Mech Eng H 224, 171-191.

19. Tinkov, S., Bekeredjian, R., Winter, G., and Coester, C. (2009) Microbubbles as Ultrasound Triggered Drug Carriers, Journal of Pharmaceutical Sciences 98, 1935-1961.

20. Dayton, P. A., and Rychak, J. J. (2007) Molecular ultrasound imaging using microbubble contrast agents, Front Biosci 12, 51245142.

21. Klibanov, A. L., Rychak, J. J., Yang, W. C., Alikhani, S., Li, B., Acton, S., Lindner, J. R., Ley, K., and Kaul, S. (2006) Targeted ultrasound contrast agent for molecular imaging of inflammation in high-shear flow, Contrast Media Mol I 1, 259-266.

22. Unger, E. C., Porter, T., Culp, W., Labell, R., Matsunaga, T., and Zutshi, R. (2004) Therapeutic applications of lipid-coated microbubbles, Advanced Drug Delivery Reviews 56, 1291-1314.

23. Finnoy, A. (2013) Acoustic and Mechanical Properties of Microbubbles Stabilized by Polymeric Nanoparticles, In Department of Physics, Norwegian University of Science and Technology, Trondheim.

24. Gresho, P. M., Sani, R. L., and Engelman, M. S. (1998) Incompressible flow and the finite element method: advection-diffusion and isothermal laminar flow, Wiley, Chichester.

25. Fung, Y. C. (1984) Biodynamics : circulation, Springer-Verlag, New York.

26. Box, F. M. A., van der Geest, R. J., Rutten, M. C. M., and Reiber, J. H. C. (2005) The influence of flow, vessel diameter, and non-Newtonian blood viscosity on the wall shear stress in a carotid bifurcation model for unsteady flow, Invest Radiol 40, 277294.

27. Sboros, V., Glynos, E., Ross, J. A., Moran, C. M., Pye, S. D., Butler, M., McDicken, W. N., Brown, S. B., and Koutsos, V. (2010) Probing microbubble targeting with atomic force microscopy, Colloid Surface B 80, 12-17.

28. Saffman, P. G. (1965) Lift on a Small Sphere in a Slow Shear Flow, Journal of Fluid Mechanics 22, 385.

29. Ward, M. D., Dembo, M., and Hammer, D. A. (1994) Kinetics of Cell Detachment — Peeling of Discrete Receptor Clusters, Biophys J 67, 2522-2534.

\section{ABOUT THE STUDENT AUTHOR}

Joshua Gosney received his A.S. degree in economics from Flathead Valley Community College in 2010. In 2012, Mr. Gosney began his academic pursuits at Montana State University, where he completed his B.S. degree in Chemical Engineering on May, 2015. Joshua Gosney has since begun his Chemical Engineering PhD. studies at the University of Arizona. At the time this paper was written, Mr. Gosney was conducting research in the computational bio-fluids laboratory under the advisement of Dr. Jeffrey Heys, and their work was focused around developing a targeted drug delivery system with use of microbubble conjugates. Mr. Gosney is a proud Native American, McNair Scholar, and an active member of the American Indian Science and Engineering Society, American Institute of Chemical Engineers student group, U.S. Green Building Council, Sustainability committee, Leadership Institute and the Undergraduate Scholars Program.

\section{SUMMARY}

The inability to noninvasively detect some diseases such as biofilm-based infections and cancer is a significant challenge. The combination of targeted microbubbles and ultrasound has been shown to potentially be an effective and inexpensive method of detection for diseases such as biofilm-based infections, cancer, and the formation of atherosclerotic plaque, among others. This study focuses on determining the biological flow conditions where microbubbles are likely to be an effective targeting agent in the human body versus flow conditions where microbubble attachment is unlikely due to large hydrodynamic forces on the microbubble. The goals of this project were to develop a numerical model predicting the forces on a microbubble, validate the model using published experimental measurements, assess the potential for using a simplified analytical model for predicting the hydrodynamic forces, and, finally, assess the potential for microbubble attachment under various biological flow conditions within the human body. 